\title{
Debromodispacamides $B$ and D: Isolation from the marine sponge Agelas mauritiana and stereoselective synthesis using a biomimetic proline route
}

\section{Carine Vergne, ${ }^{\dagger}$ Jérôme Appenzeller,${ }^{\dagger}$ Céline Ratinaud, ${ }^{\dagger}$ Marie-Thérèse Martin, ${ }^{\dagger}$ Cécile Debitus, ${ }^{\ddagger}$ Anne Zaparucha $^{\dagger}$ and Ali Al-Mourabit ${ }^{\star, \dagger}$}

${ }^{\dagger}$ Institut de Chimie des Substances Naturelles du CNRS, Avenue de la Terrasse, 91198 Gif-sur-Yvette, France ${ }^{*}$ UMR 152 IRD-Université Paul Sabatier Toulouse III, Faculté des Sciences Pharmaceutiques, 31062 Toulouse cedex 9, France

I. General Experiment Procedures

II. Natural metabolites isolation

III. Synthetic experimental part

IV. ${ }^{1} \mathrm{H}$ and ${ }^{13} \mathrm{C}$ NMR spectra for all new compounds

\section{General Experiment Procedures:}

NMR spectra were recorded on Spectrometers $300 \mathrm{MHz}$ and $500 \mathrm{MHz}$ Bruker for ${ }^{1} \mathrm{H}$ and ${ }^{13} \mathrm{C}$ NMR and $600 \mathrm{MHz}$ Bruker for NOESY and 2D NMR experiments. Chemical shifts (expressed in ppm) of ${ }^{1} \mathrm{H}$ and ${ }^{13} \mathrm{C}$ NMR spectra were referenced to the solvent peaks $\delta_{\mathrm{H}} 3.31$ and $\delta_{\mathrm{C}} 49.1$ for $\mathrm{CD}_{3} \mathrm{OD}, \delta_{\mathrm{H}}$ 2.50 and $\delta_{\mathrm{C}} 39.5$ for DMSO-d6. HRMS mass spectra were obtained with an electrospray source (Lockspray) coupled with a time of flight analyser (LCT, Micromass). Samples were prepared in methanol and injected in the MS system using a Waters 2795 system. The mobile phase was $\mathrm{MeOH} /$ water $(50 / 50, \mathrm{v} / \mathrm{v})$ with a $0.2 \mathrm{~mL} / \mathrm{min}$ flow. Preparative HPLC were performed on an autoprep system (Waters 600 controller and Waters 600 pump with a Waters 996 PhotoDiode Array detector). Samples were injected by the Waters 2700 Sample Manager. Waters XBridge Prep Shield RP18 $5 \mu \mathrm{m} \mathrm{19*150} \mathrm{mm} \mathrm{and} \mathrm{Waters} \mathrm{Atlantis} \mathrm{Hilic} \mathrm{Silica} 5 \mu \mathrm{m} \mathrm{10*150} \mathrm{mm} \mathrm{columns} \mathrm{were} \mathrm{used} \mathrm{for}$ preparative HPLC. Waters XBridge RP18 5um 3.0*150 mm and Waters Atlantis Hilic Silica $5 \mu \mathrm{m}$ 4.6*150 mm columns were used for analysis. Optical rotations were measured on a Jasco P-1010 
polarimeter in $\mathrm{MeOH}$. IR spectra were acquired (neat) using a Perkin Elmer Spectrometer BX FTIR system.

\section{Natural metabolites isolation:}

\section{- Animal Material.}

The sponge Agelas mauritiana. (Order Agelasida, Family Agelasidae) was collected by scuba at depth between 18 to $30 \mathrm{~m}$ on Guadalcanal reefs off the Solomon Islands in June 2004. The specimens were immediately frozen at $-80^{\circ} \mathrm{C}$ and lyophilized. A voucher specimen is deposited in the Centre Oceanologique de Marseille by N. Boury-Esnault, under the accessing number IRDR3016.

\section{- Extraction and Isolation.}

The sponge dry powder (540 g) was extracted three times overnight with dichloromethane (3x3 L) and then with methanol $(3 \times 3 \mathrm{~L})$. Solvents were evapored under reduced pressure and the resulting crude methanolic extract $(50 \mathrm{~g})$ was partitioned between $n$ - $\mathrm{BuOH}(50 \mathrm{~mL}$ x 10$)$ and $\mathrm{H}_{2} \mathrm{O}(700 \mathrm{~mL})$. The $n$ - $\mathrm{BuOH}$ soluble materials (30 g) was chromatographed (20 g) on a silica column (Versaflash Supelco, 20-45 $\mu \mathrm{m}$ spherical silica, $80 \times 150 \mathrm{~mm}$ ) with a dichloromethane/methanol gradient as eluent, monitored by TLC. Alkaloid-containing fraction $(450 \mathrm{mg}$ ) eluated with $15 \% \mathrm{MeOH}$ was purified further by preparative HPLC using a preparative $\mathrm{C}_{18}$ column $\left(\mathrm{XBridge}, \mathrm{CH}_{3} \mathrm{CN} /\right.$ water + $\mathrm{AcONH}_{4}-10 \mathrm{mmol} \mathrm{pH}=9,5-0 / 100$ to $20 / 80$ in $50 \mathrm{~min}$, flow rate $17 \mathrm{ml} / \mathrm{min}$, UV detection at 260 $\mathrm{nm})$ to yield debromodispacamide $\mathrm{B}(\mathbf{1})\left(\mathrm{t}_{\mathrm{R}}=17.7 \mathrm{~min} ; 3 \mathrm{mg}\right.$; yield: $0.0006 \%$, dry wt $)$ and debromodispacamide D (2) $\left(\mathrm{t}_{\mathrm{R}}=12.5 \mathrm{~min} ; 3 \mathrm{mg}\right.$; yield: 0.0006\%, dry wt).

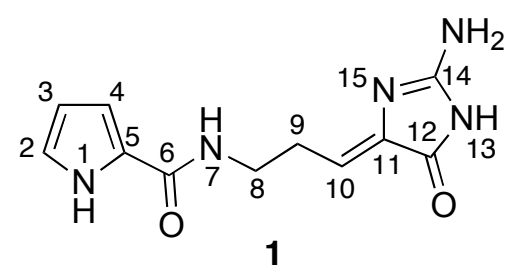

Debromodispacamide B (1): pale yellow solid; ${ }^{1} \mathrm{H}$ NMR $\left(\mathrm{CD}_{3} \mathrm{OD}, 300 \mathrm{MHz}\right) \delta 6.86(1 \mathrm{H}, \mathrm{dd}, J=$ 2.5 and $1.4 \mathrm{~Hz}, \mathrm{H}-2), 6.72(1 \mathrm{H}, \mathrm{dd}, J=3.9$ and $1.4 \mathrm{~Hz}, \mathrm{H}-4), 6.11(1 \mathrm{H}, \mathrm{dd}, J=3.9$ and $2.5 \mathrm{~Hz}, \mathrm{H}-3)$, $5.71(1 \mathrm{H}, \mathrm{t}, J=7.9 \mathrm{~Hz}, \mathrm{H}-10), 3.41(1 \mathrm{H}, \mathrm{t}, J=6.9 \mathrm{~Hz}, \mathrm{H}-8), 2.49(2 \mathrm{H}, \mathrm{dd}, J=7.9$ and $6.9 \mathrm{~Hz}, \mathrm{H}-9)$; 
${ }^{13} \mathrm{C}$ NMR (CD $\left.\mathrm{OD}, 75 \mathrm{MHz}\right)$ o 28.9 (C9), 39.6 (C8), 110.4 (C10), 110.4 (C3), 111.9 (C4), 123.1 (C2), 126.9 (C5), 136.9 (C11), 164.1 (C6), 168.4 (C12), 179.3 (C14); IR (neat) v $v_{\max }$ 3162, 1715, 1614, 1556 and $1458 \mathrm{~cm}^{-1}$; UV (MeOH) $\lambda_{\max } 252 \mathrm{~nm}$; MS (ESI) m/z $248.1[\mathrm{M}+\mathrm{H}]^{+}, 270.2[\mathrm{M}+\mathrm{Na}]^{+}$ HRMS calcd. for $\mathrm{C}_{11} \mathrm{H}_{14} \mathrm{~N}_{5} \mathrm{O}_{2}[\mathrm{M}+\mathrm{H}]^{+} 248.1147$, found 248.1122.

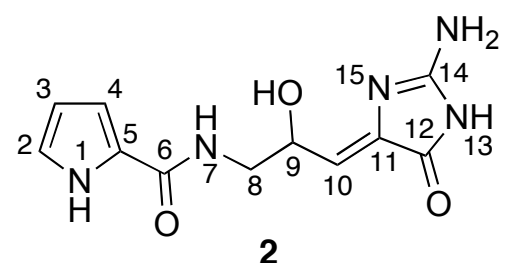

Debromodispacamide D (2): yellow solid; $[\alpha]^{25} 0$ (c 0.28, MeOH); ${ }^{1} \mathrm{H}$ NMR $\left(\mathrm{CD}_{3} \mathrm{OD}, 500 \mathrm{MHz}\right)$ $\delta_{\mathrm{H}} 6.93(1 \mathrm{H}, \mathrm{dd}, J=2.5$ and $1.3 \mathrm{~Hz}, \mathrm{H}-2), 6.80(1 \mathrm{H}, \mathrm{dd}, J=3.8$ and $1.3 \mathrm{~Hz}, \mathrm{H}-4), 6.18(1 \mathrm{H}, \mathrm{dd}, J=$ 3.8 and $2.5 \mathrm{~Hz}, \mathrm{H}-3), 5.72(1 \mathrm{H}, \mathrm{d}, J=5.3 \mathrm{~Hz}, \mathrm{H}-10), 4.67$ (1H, m, H-9), 3.49 (2H, m, H-8); ${ }^{13} \mathrm{C}$ NMR (DMSO-d6, 300 MHz) $\delta_{\mathrm{C}} 44.9$ (C8), 67.3 (C9), 108.5 (C3), 109.3 (C10, HSQC), 110.1 (C4), 121.3 (C2), 126.1 (C5), 134.5 (C11, HMBC), 160.9 (C6), 177.2 (C12, HMBC); IR (neat) $v_{\max } 3285$, 1692, 1626, 1565 and $1529 \mathrm{~cm}^{-1}$; UV (MeOH) $\lambda_{\max }$ 265, $322 \mathrm{~nm}$; MS (ESI) m/z $264.1[\mathrm{M}+\mathrm{H}]^{+}$, $286.1[\mathrm{M}+\mathrm{Na}]^{+}$; HRMS calcd. for $\mathrm{C}_{11} \mathrm{H}_{13} \mathrm{~N}_{5} \mathrm{O}_{3} \mathrm{Na}[\mathrm{M}+\mathrm{Na}]^{+}$286.0916, found 286.0907.

\section{Synthetic experimental part:}

Debromodispacamide B (1): DMF (12.5 mL) was added to a pre-dried (vacuum pump, $30 \mathrm{~min}$ ) mixture of guanidine carbonate (360 mg, 2 mmol., 2 equiv.), pseudo peptide pyrrole-proline methyl ester 10 (222 mg, $1 \mathrm{mmol}$.) and crushed $4 \AA$ molecular sieves $(1.2 \mathrm{~g})$. The reaction mixture was stirred at $90{ }^{\circ} \mathrm{C}$ for 45 minutes then cooled in an ice bath. After filtration of the reaction mixture, the solvent was evaporated in vacuo. Purification by silica gel chromatography using 80:20 $\mathrm{NH}_{3^{-}}$ saturated $\mathrm{CH}_{2} \mathrm{Cl}_{2}: \mathrm{MeOH}$ afforded $\mathbf{1}(143 \mathrm{mg}, 56 \%)$ and $\mathbf{1 0}(30 \mathrm{mg}, 12 \%)$.

\section{2-imino-6-(1H-pyrrole-2-carbonyl)-1,3,6-triazaspiro[4.4]nonan-4-one (10):}

${ }^{1} \mathrm{H}$ NMR $\left(\mathrm{CD}_{3} \mathrm{OD}, 300 \mathrm{MHz}\right) \delta_{\mathrm{H}} 7.01(\mathrm{~m}, 1 \mathrm{H}), 6.81(\mathrm{~m}, 1 \mathrm{H}), 6.25(\mathrm{~m}, 1 \mathrm{H}), 4.06(\mathrm{~m}, 1 \mathrm{H}), 3.93(\mathrm{~m}$, $1 \mathrm{H}), 2.26(\mathrm{~m}, 1 \mathrm{H}) ;{ }^{13} \mathrm{C}\left(\mathrm{CD}_{3} \mathrm{OD}, 75 \mathrm{MHz}\right) \delta_{\mathrm{C}} 25.1,37.4,50.7,81.7,111.0,115.6,124.5,162.4 ; \mathrm{IR}$ $(\mathrm{NaCl}) v_{\max } 3306,1722,1595$ and $1427 \mathrm{~cm}^{-1}$; MS (ESI) $m / z 248.1[\mathrm{M}+\mathrm{H}]^{+}, 270.1[\mathrm{M}+\mathrm{Na}]^{+}$; HRMS calcd. for $\mathrm{C}_{11} \mathrm{H}_{14} \mathrm{~N}_{5} \mathrm{O}_{2}[\mathrm{M}+\mathrm{H}]^{+}$248.1148, found 248.1147.

(R)-Debromodispacamide D (2): DMF (12 mL) was added to a pre-dried (vacuum pump, $30 \mathrm{~min}$ ) mixture of guanidine carbonate (360 mg, 2 mmol., 2 equiv.), pseudo peptide pyrrole-hydroxyproline methyl ester 13 (238 mg, 1 mmole) and crushed $4 \AA$ molecular sieves (1.2 g). The reaction mixture was stirred at $90{ }^{\circ} \mathrm{C}$ for 30 minutes then cooled in an ice bath. After filtration of the reaction 
mixture, the solvent was evaporated in vacuo. Purification by silica gel chromatography using 90:10 $\mathrm{NH}_{3}$-saturated $\mathrm{CH}_{2} \mathrm{Cl}_{2}-\mathrm{MeOH}$ afforded 2 (70 mg, 27\%, yellow solid; $[\alpha]{ }^{25} 41.7(\mathrm{c}=0.34$, $\mathrm{MeOH})$ ) and $15\left(5 \mathrm{mg}, 2 \%\right.$ ) after final purification by $\mathrm{RP}_{18} \mathrm{HPLC}$ (Hilic, $\mathrm{CH}_{3} \mathrm{CN} /$ water + $\mathrm{HCOONH}_{4}-100 \mathrm{mmol} \mathrm{pH}=3.8 /$ water $-95 / 5 / 0$ to $60 / 5 / 35$ in $60 \mathrm{~min}$, flow rate $5 \mathrm{ml} / \mathrm{min}$, UV detection at $260 \mathrm{~nm}$ ).

(8R)-8-hydroxy-2-imino-6-(1H-pyrrole-2-carbonyl)-1,3,6-triazaspiro[4.4]nonan-4-one (15) : white solid; $[\alpha]{ }_{\mathrm{D}}^{25} 14\left(\mathrm{c}=0.13, \mathrm{CH}_{3} \mathrm{OH}\right) ;{ }^{1} \mathrm{H}$ NMR $\left(\mathrm{CD}_{3} \mathrm{OD}, 300 \mathrm{MHz}\right) \delta_{\mathrm{H}} 6.98(\mathrm{dd}, 2.4,1.2,1 \mathrm{H})$, $6.74(\mathrm{dd}, 4.0,1.2,1 \mathrm{H}), 6.23(\mathrm{dd}, 4.0,2.4,1 \mathrm{H}), 4.62(\mathrm{~m}, 1 \mathrm{H}), 4.04(\mathrm{dd}, 10.4,3.8,1 \mathrm{H}), 3.96$ (brd, 10.4, 1H), $2.33(\mathrm{dd}, 13.0,3.6,1 \mathrm{H}), 2.09(\mathrm{~m}, 1 \mathrm{H}) ;{ }^{13} \mathrm{C}$ (DMSO-d6, 300MHz) $\delta_{\mathrm{C}} 44.3,57.4,68.4$, 79.7, 109.1, 112.3, 122.0, 125.3, 158.8, 170.0, 186.2; ); IR (neat) $v_{\max } 3279,1650,1643,1592,1574$, 1556, 1427, 1337, 1194 and $1043 \mathrm{~cm}^{-1}$; UV (MeOH) $\lambda_{\max } 268 \mathrm{~nm}$; MS (ESI) m/z $264.2[\mathrm{M}+\mathrm{H}]^{+}$; HRMS calcd. for $\mathrm{C}_{11} \mathrm{H}_{12} \mathrm{~N}_{5} \mathrm{O}_{3}[\mathrm{M}-\mathrm{H}]^{-}$262.0940, found 262.0942.

(2S,4R)-methyl-4-hydroxy-1-(1H-pyrrole-2-carbonyl)pyrrolidine-2-carboxylate (13); (3R,5S)5-(methoxycarbonyl)-1-(1H-pyrrole-2-carbonyl)pyrrolidin-3-yl $1 H$-pyrrole-2-carboxylate (14): EDCI (3.6 g, 18.72 mmol., 2 equiv.) was added to a solution of pyrrole-2-carboxylic acid (1.04g, 9.36 mmoles), trans-L-4-hydroxyproline methyl ester and DMAP (catalytic) in dichloromethane (30 $\mathrm{mL}$ ) under Argon atmosphere at $0{ }^{\circ} \mathrm{C}$. The reaction mixture was stirred for $2 \mathrm{~h}$ at $\mathrm{RT}$ and for $2 \mathrm{~h}$ under reflux. After addition of brine, the organic layer was extracted twice with AcOEt then with $n$ $\mathrm{BuOH}$. The organic layers were dried over MgSO4 and the solvents were evaporated in vacuo. Purification by silica gel chromatography using 65:35 to 90:10 heptane:AcOEt gradient, afforded 13 (1.59 g, 71\%) and $14(0.13 \mathrm{~g}, 9 \%)$.

13: white foam; $[\alpha]_{\mathrm{D}}^{25}-32.7\left(\mathrm{c}=0.50, \mathrm{CHCl}_{3}\right) ;{ }^{1} \mathrm{H} \mathrm{NMR}\left(\mathrm{CDCl}_{3}, 300 \mathrm{MHz}\right) \delta_{\mathrm{H}} 10.74(\mathrm{~s}, 1 \mathrm{H}), 6.87$ $(\mathrm{m}, 1 \mathrm{H}), 6.52(\mathrm{~m}, 1 \mathrm{H}), 6.16(\mathrm{~m}, 1 \mathrm{H}), 4.72(\mathrm{t}, J=7.91 \mathrm{~Hz}, 1 \mathrm{H}), 4.33(\mathrm{~m}, 1 \mathrm{H}), 4.45(\mathrm{~m}, 1 \mathrm{H}), 3.81(\mathrm{~m}$, 2H), $3.65(\mathrm{~s}, 3 \mathrm{H}), 2.21(\mathrm{~m}, 1 \mathrm{H}), 1.93(\mathrm{~m}, 1 \mathrm{H}) ;{ }^{13} \mathrm{C}\left(\mathrm{CDCl}_{3}, 75 \mathrm{MHz}\right) \delta_{\mathrm{C}} 37.0,52.3,56.8,59.0,70.6$, 109.9, 113.7, 122.5, 124.4, 161.5, 173.3; IR (neat) $v_{\max } 3600-3100,1731,1580,1546,1433$; MS (ESI) $m / z 239.1[\mathrm{M}+\mathrm{H}]^{+}, 261.1[\mathrm{M}+\mathrm{Na}]^{+}$; HRMS calcd. for $\mathrm{C}_{11} \mathrm{H}_{14} \mathrm{~N}_{2} \mathrm{O}_{4} \mathrm{Na}[\mathrm{M}+\mathrm{Na}]^{+} 261.0851$, found 261.0838 .

14: white solid; $[\alpha]^{24}-54.7\left(\mathrm{c}=0,40, \mathrm{CHCl}_{3}\right) ;{ }^{1} \mathrm{H} \mathrm{NMR}\left(\mathrm{CDCl}_{3}, 300 \mathrm{MHz}\right) \delta_{\mathrm{H}} 10.44(\mathrm{~s}, 1 \mathrm{H}), 9.94$ $(\mathrm{s}, 1 \mathrm{H}), 6.93(\mathrm{~m}, 2 \mathrm{H}), 6.88(\mathrm{~m}, 1 \mathrm{H}), 6.61(\mathrm{~m}, 1 \mathrm{H}), 6.21(\mathrm{~m}, 2 \mathrm{H}), 5.64(\mathrm{~m}, 1 \mathrm{H}), 4.85(\mathrm{t}, J=8.19 \mathrm{~Hz}$, $1 \mathrm{H}), 4.22(\mathrm{~m}, 1 \mathrm{H}), 4.10(\mathrm{~m}, 1 \mathrm{H}), 3.71(\mathrm{~s}, 3 \mathrm{H}), 2.52(\mathrm{~m}, 1 \mathrm{H}), 2.28(\mathrm{~m}, 1 \mathrm{H}) ;{ }^{13} \mathrm{C} \mathrm{NMR}\left(\mathrm{CDCl}_{3}\right.$, $75 \mathrm{MHz}) \delta_{\mathrm{C}} 34.6,52.6,54.4,59.2,73.2,110.3,110.7,113.6,116.6,121.7,122.6,124.2,124.7$, 
160.6, 161.2, 172.6; IR (neat) vmax 3200, 1748, 1692, 1591, 1546, 1436; MS (ESI) $\mathrm{m} / z$ 354.1 $[\mathrm{M}+\mathrm{Na}]^{+}$; HRMS calcd. for $\mathrm{C}_{16} \mathrm{H}_{17} \mathrm{~N}_{3} \mathrm{O}_{5} \mathrm{Na}[\mathrm{M}+\mathrm{Na}]^{+}$354.1066, found 354.1076.

$(Z)$ and (E)-oxazoline (17a+17b): Debromodispacamide D (2) (50 mg, 0.19 mmol.) was dissolved in TFA $(2 \mathrm{~mL})$ and the resulting solution heated at $60^{\circ} \mathrm{C}$ for 2 hours. The solvent was removed under reduced pressure. The resulting crude was purified by $\mathrm{RP}_{18} \mathrm{HPLC}$ (Hilic, $\mathrm{CH}_{3} \mathrm{CN} /$ water + $\mathrm{HCOONH}_{4}-100 \mathrm{mmol}$. $\mathrm{pH}=3.8 /$ water $-95 / 5 / 0$ to $60 / 5 / 35$ in $60 \mathrm{~min}$, flow rate $5 \mathrm{ml} / \mathrm{min}$, UV detection at $260 \mathrm{~nm}$ ) to yield a nearly 1:1 mixture of $\mathbf{1 7 a}$ and $\mathbf{1 7 b}(10 \mathrm{mg}, 0.04 \mathrm{mmol}, 21 \%)$ as formates. By heating this mixture in $\mathrm{CH}_{3} \mathrm{OH} / \mathrm{HCl} 1 \mathrm{~N} \mathrm{2:1}$ at $60^{\circ} \mathrm{C}$ for 2.5 hours, 17a was obtained as the major diastereoisomer $(\mathbf{1 7} \mathbf{a}+\mathbf{1 7} \mathbf{b}>10: 1)$ in quantitative yield as chlorhydrate.

(Z)-oxazoline (17a): ${ }^{1} \mathrm{H}$ NMR $\left(\mathrm{CD}_{3} \mathrm{OD}, 500 \mathrm{MHz}\right) \delta_{\mathrm{H}} 6.92(1 \mathrm{H}, \mathrm{m}, \mathrm{H}-2), 6.78(1 \mathrm{H}, \mathrm{m}, \mathrm{H}-4), 6.16$ $(1 \mathrm{H}, \mathrm{m}, \mathrm{H}-3), 5.64$ (1H, d, J = $6.7 \mathrm{~Hz}, \mathrm{H}-10), 4.26$ (1H, m, H-9), 3.52 (2H, m, H-8); ${ }^{13} \mathrm{C} \mathrm{NMR}$ $\left(\mathrm{CD}_{3} \mathrm{OD}, 500 \mathrm{MHz}\right) \delta_{\mathrm{C}} 43.8(\mathrm{C} 8), 78.4(\mathrm{C} 9), 110.3$ (C3), 111.0 (C10), 112.0 (C4), 123.1 (C2), 126.8 (C5), 136.4 (C11), 164.0 (C6), 167.2 (formiate), 178.0 (C12); IR (neat) $v_{\max }$ 1695, 1623, 1569, 1559, 1521, 1472, 1325, 1203, $1115 \mathrm{~cm}^{-1}$; UV (MeOH) $\lambda_{\max } 228,268 \mathrm{~nm} ; \mathrm{MS}$ (ESI) m/z 246.2 $[\mathrm{M}+\mathrm{H}]^{+}, 244.2[\mathrm{M}-\mathrm{H}]^{-}$; HRMS calcd for $\mathrm{C}_{11} \mathrm{H}_{12} \mathrm{~N}_{5} \mathrm{O}_{2}[\mathrm{M}+\mathrm{H}]^{+}$246.0991, found 246.0985.

(E)-oxazoline (17b): ${ }^{1} \mathrm{H}$ NMR $\left(\mathrm{CD}_{3} \mathrm{OD}, 500 \mathrm{MHz}\right) \delta_{\mathrm{H}} 6.89$ (1H, m, H-2), 6.75 (1H, m, H-4), 6.14 $(1 \mathrm{H}, \mathrm{m}, \mathrm{H}-3), 5.43$ (1H, d, J = $9.5 \mathrm{~Hz}, \mathrm{H}-10), 5.28$ (1H, m, H-9), 3.47 (2H, m, H-8); ${ }^{13} \mathrm{C}$ NMR $\left(\mathrm{CD}_{3} \mathrm{OD}, 500 \mathrm{MHz}\right) \delta_{\mathrm{C}} 44.2$ (C8), 74.3 (C9), 110.2 (C3), 111.9 (C4), 116.3 (C4), 122.9 (C2), 126.7 (C5), 137.2 (C11), 163.8 (C6), 167.2 (formate), 177.6 (C12).

\section{$\underline{\text { IV. }}{ }^{1} \mathrm{H}$ and ${ }^{13} \mathrm{C}$ NMR spectra for all new compounds}


Debromodispacamide B (1), ${ }^{1} \mathrm{H}$ NMR:

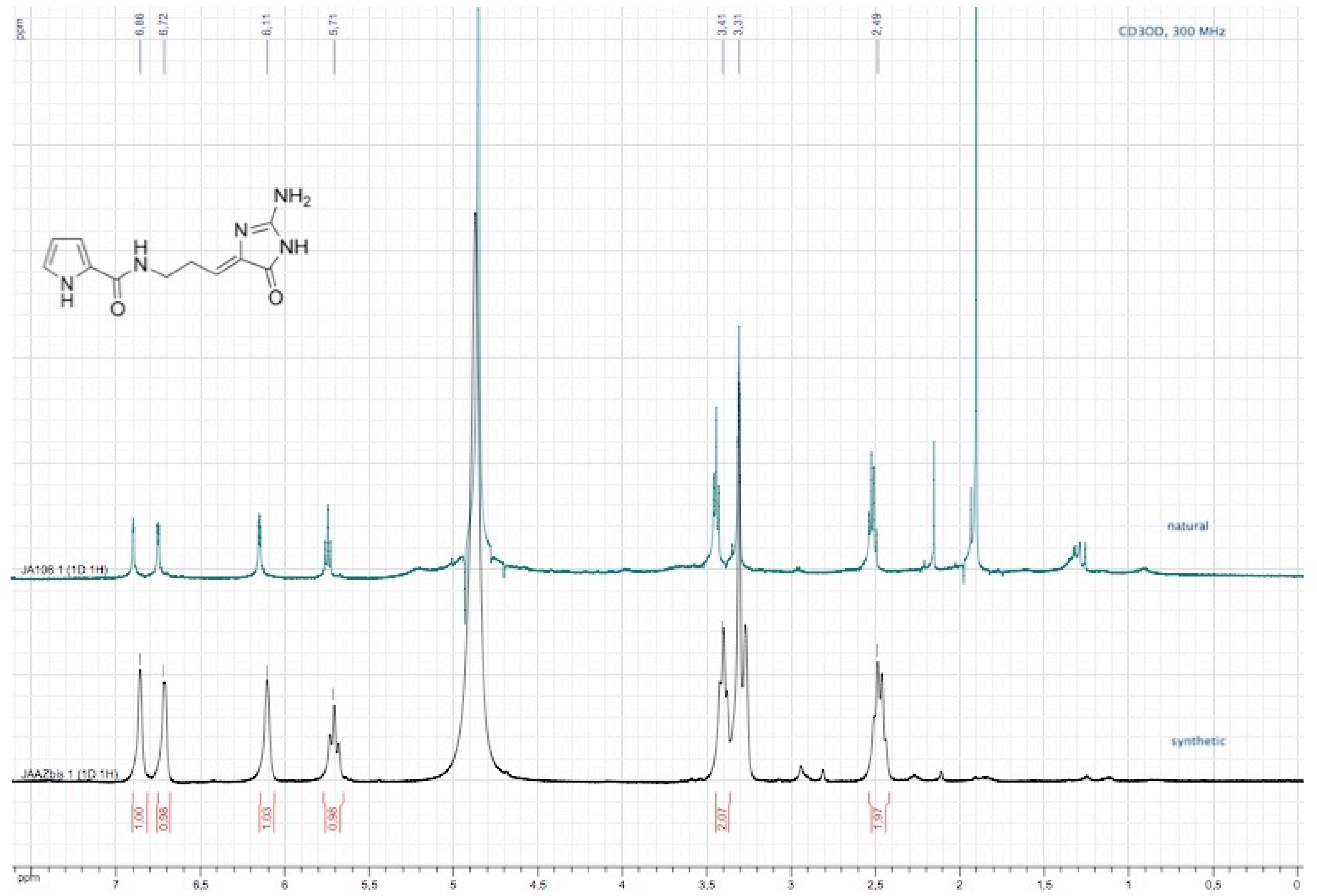


Debromodispacamide B (1) ${ }^{13} \mathrm{C}$ NMR:
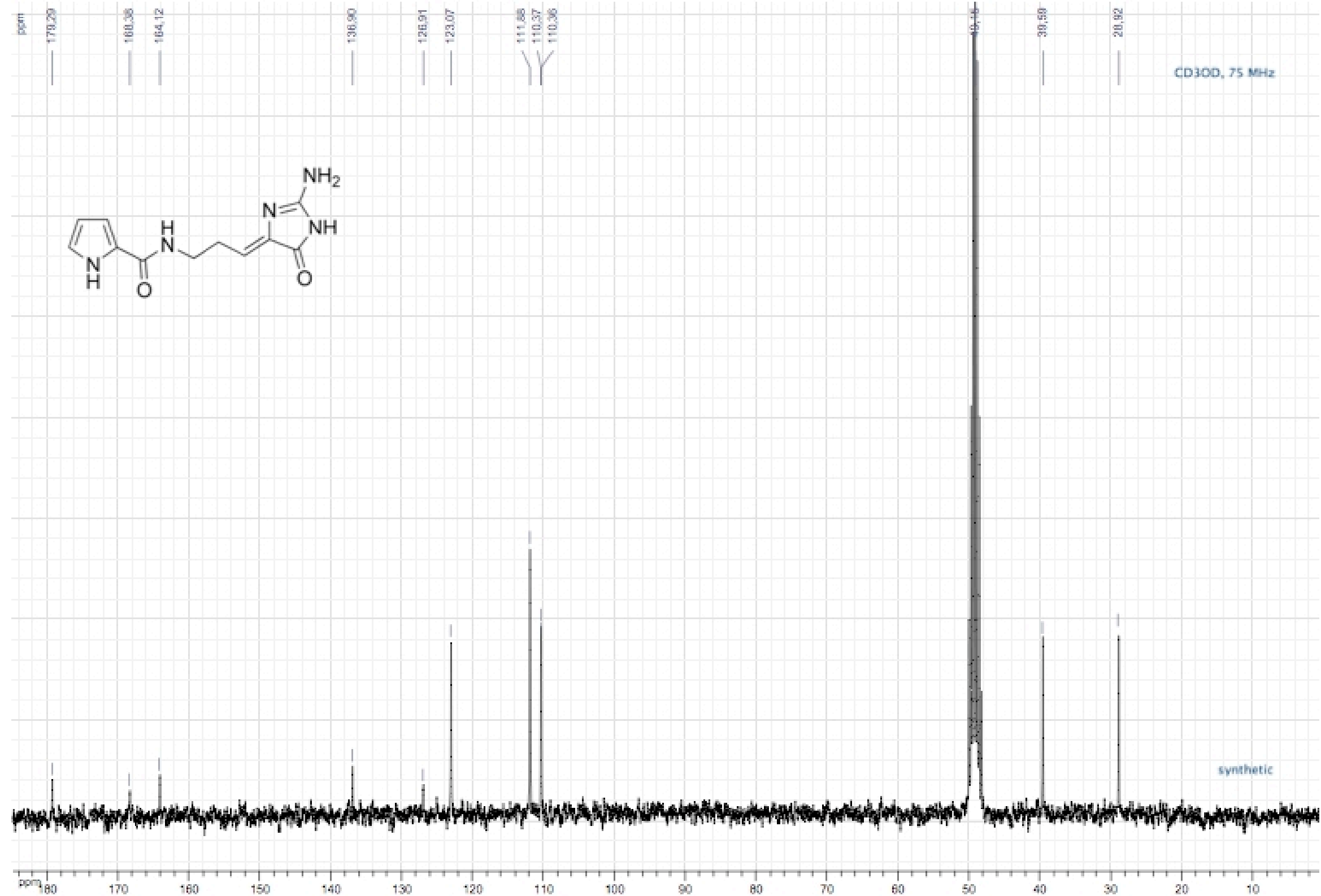
Debromodispacamide D (2), ${ }^{1} \mathrm{H}$ NMR:

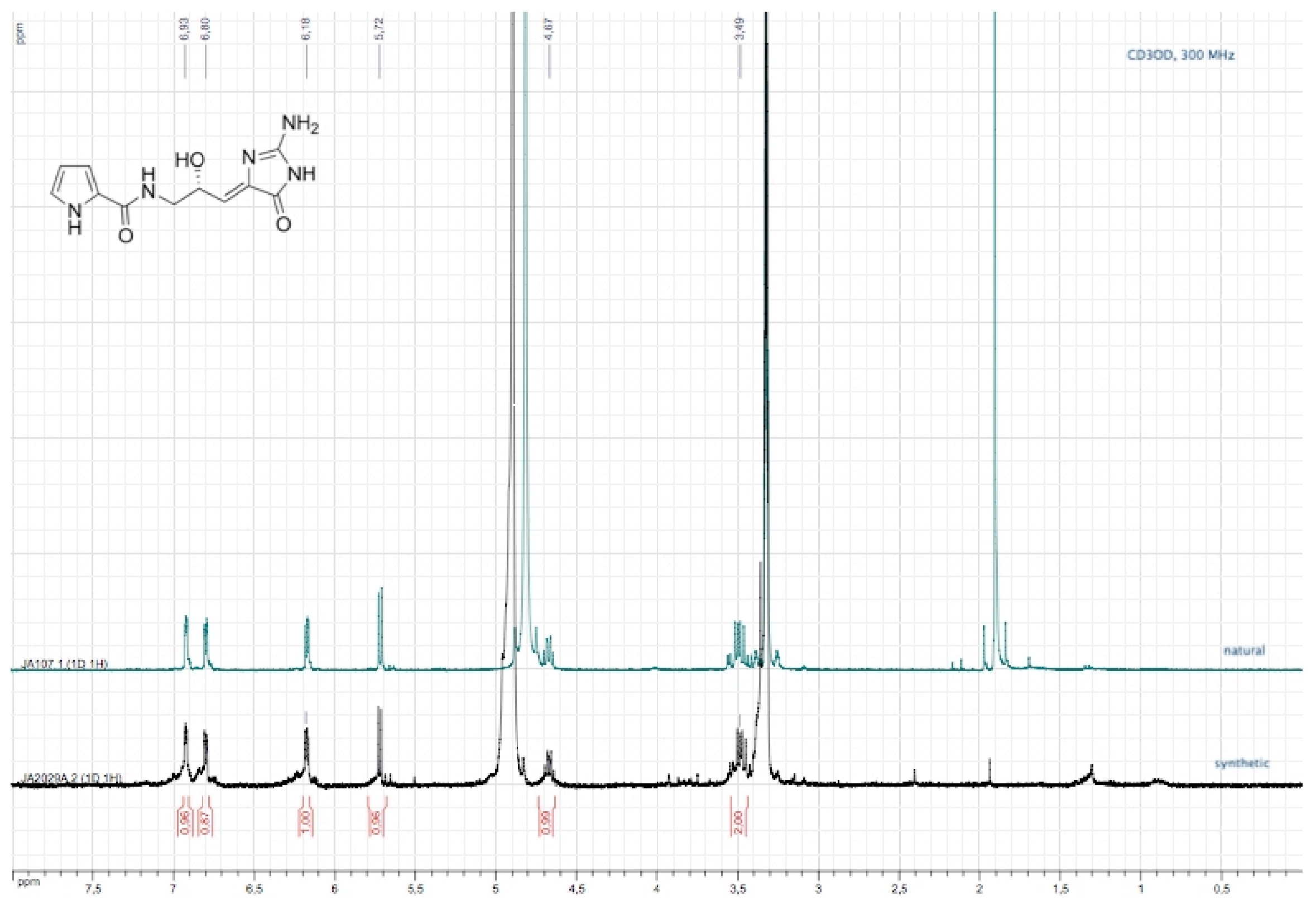


Debromodispacamide D (2), ${ }^{13} \mathrm{C}$ NMR:
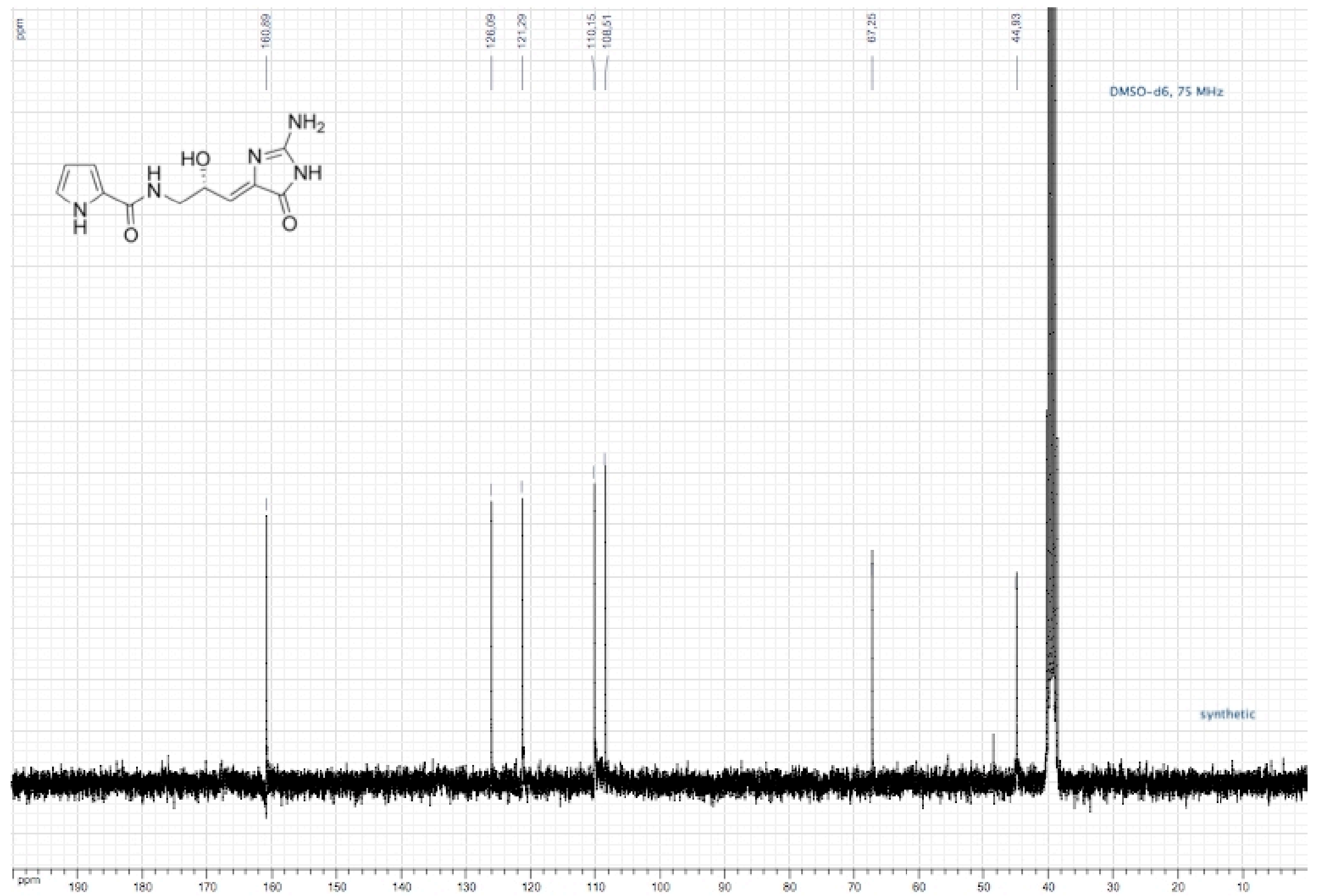
2-imino-6-(1H-pyrrole-2-carbonyl)-1,3,6-triazaspiro[4.4]nonan-4-one (10), ${ }^{1} \mathrm{H}$ NMR :

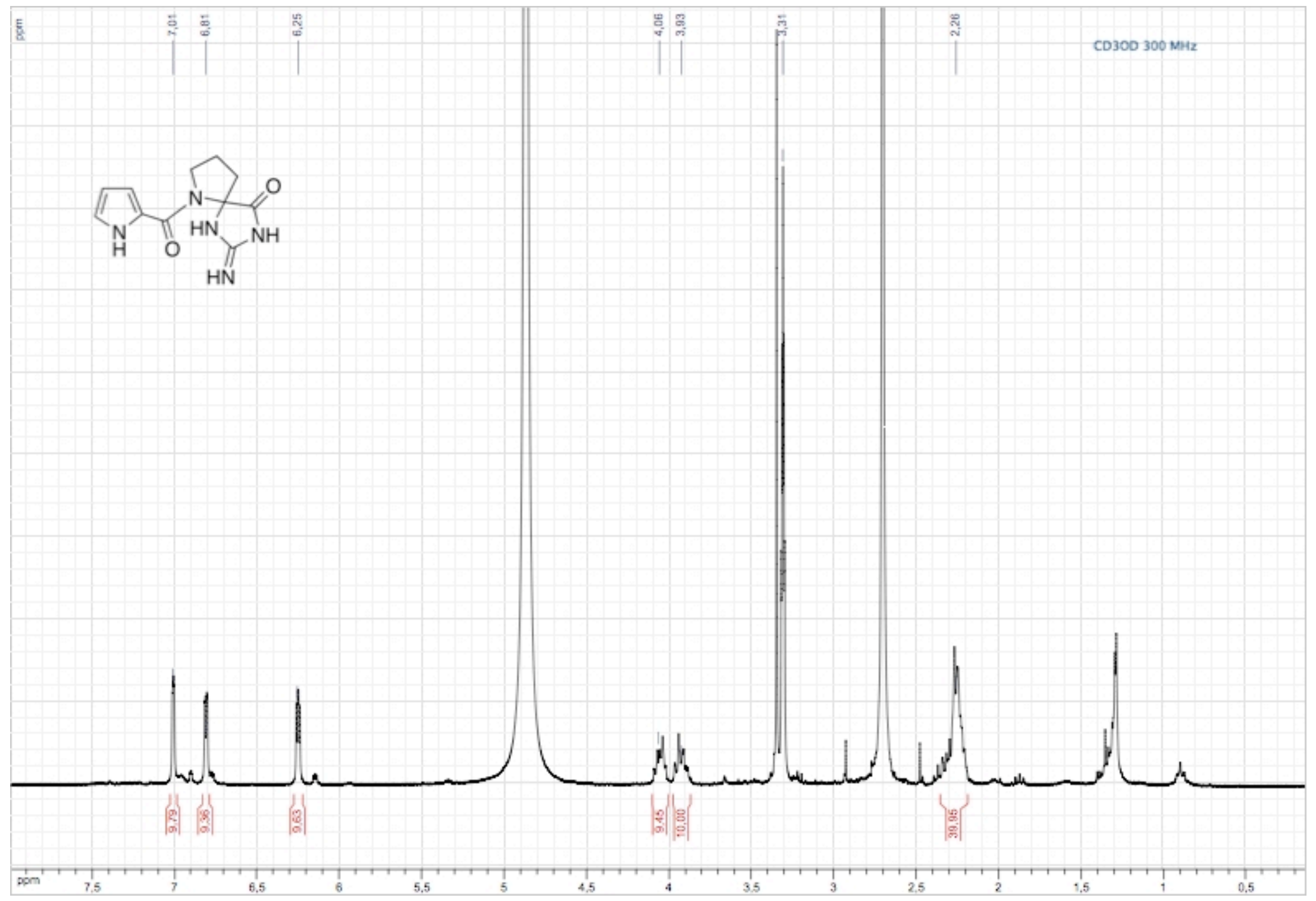


2-imino-6-(1H-pyrrole-2-carbonyl)-1,3,6-triazaspiro[4.4] nonan-4-one (10), ${ }^{13} \mathrm{C}$ NMR :
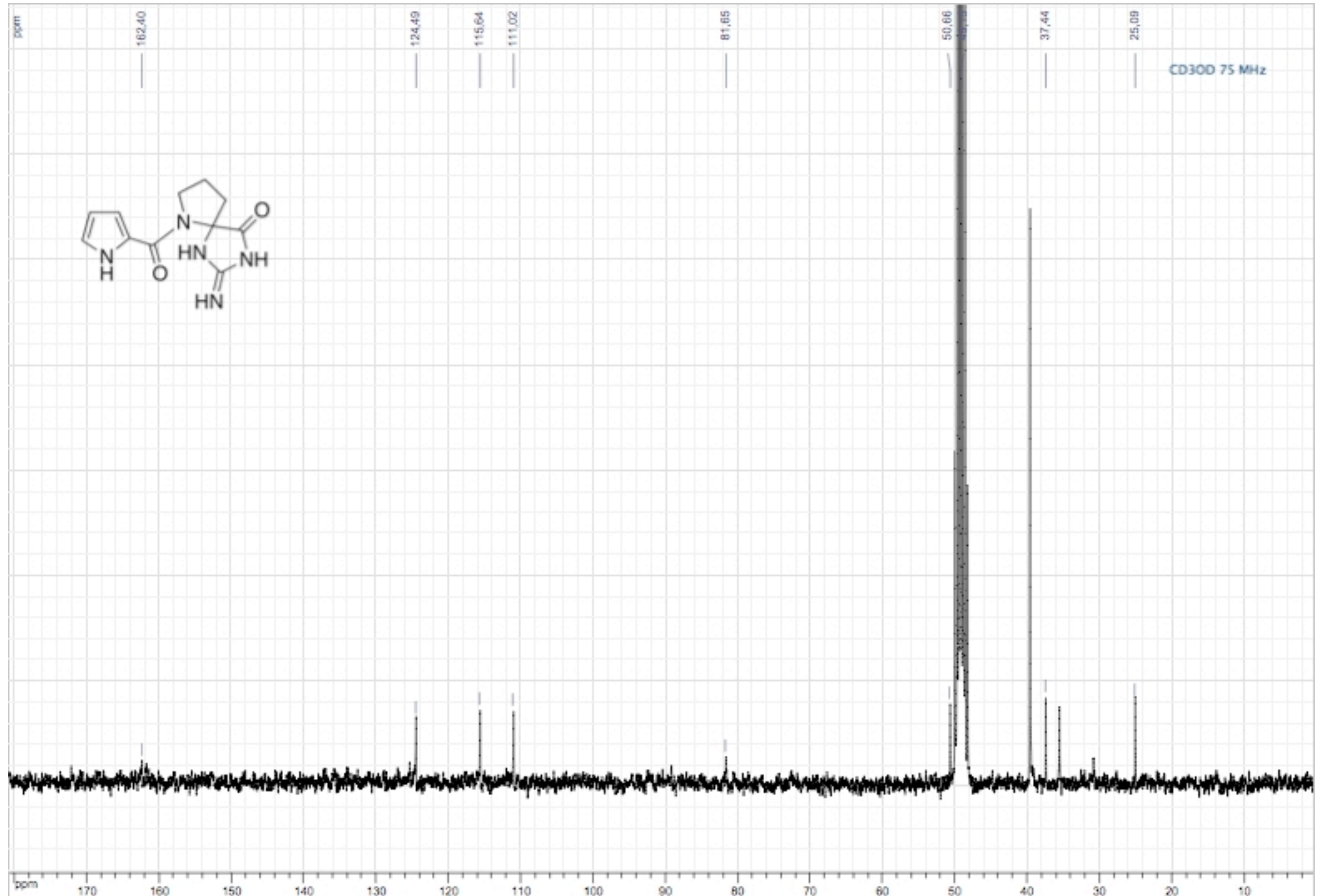

$160 \quad 150$

140

130

110

100

$\infty$

ө0

60

so

40 
(2S,4R)-methyl-4-hydroxy-1-(1H-pyrrole-2-carbonyl)pyrrolidine-2-carboxylate (13), ${ }^{1} \mathrm{H}$ NMR:

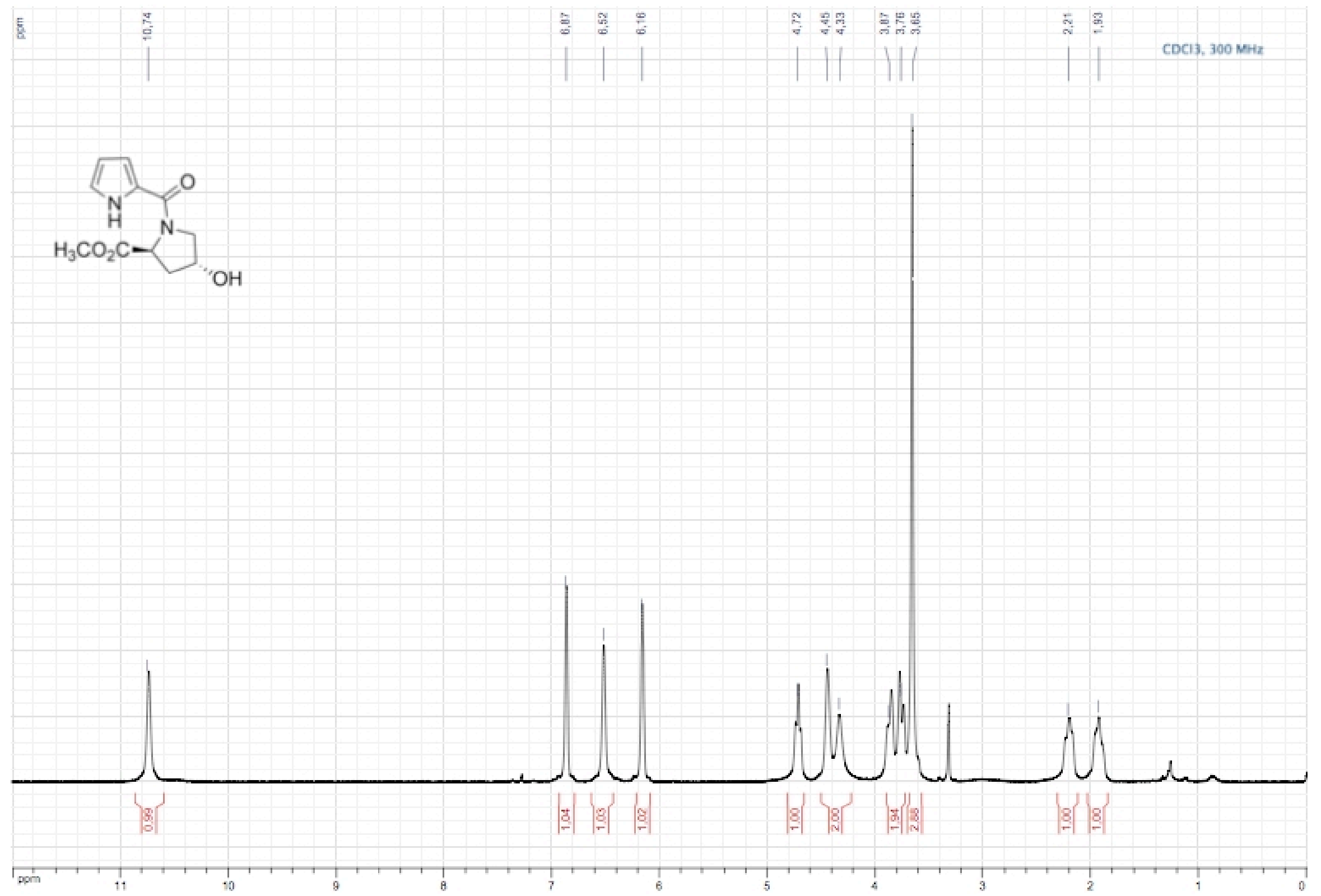


(2S,4R)-methyl-4-hydroxy-1-(1H-pyrrole-2-carbonyl)pyrrolidine-2-carboxylate (13), ${ }^{13} \mathrm{C}$ NMR:
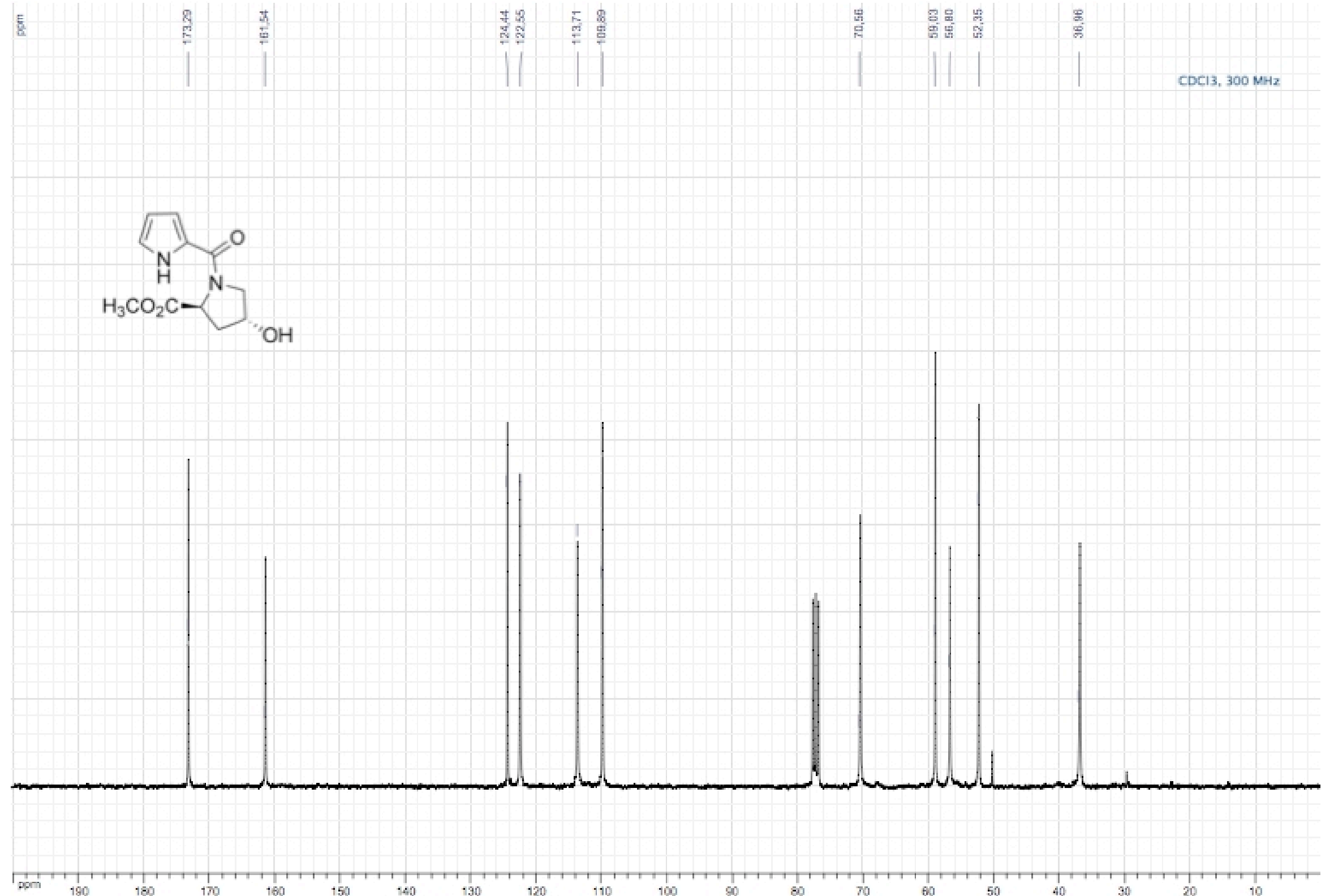
(3R,5S)-5-(methoxycarbonyl)-1-(1H-pyrrole-2-carbonyl)pyrrolidin-3-yl $1 H$-pyrrole-2-carboxylate (14), ${ }^{1} \mathrm{H}$ NMR:

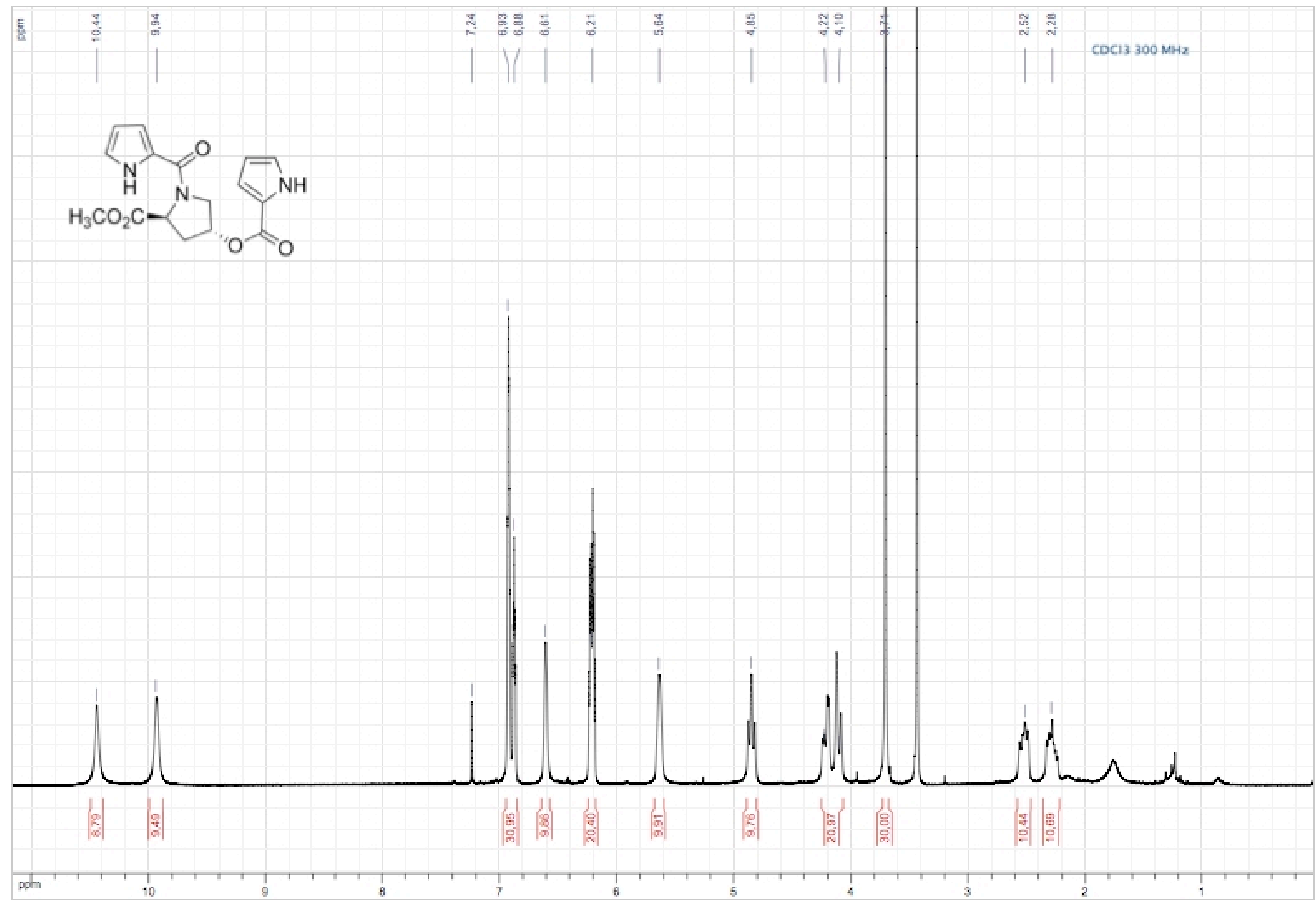


(3R,5S)-5-(methoxycarbonyl)-1-(1H-pyrrole-2-carbonyl)pyrrolidin-3-yl $1 H$-pyrrole-2-carboxylate (14), ${ }^{13} \mathrm{C}$ NMR:

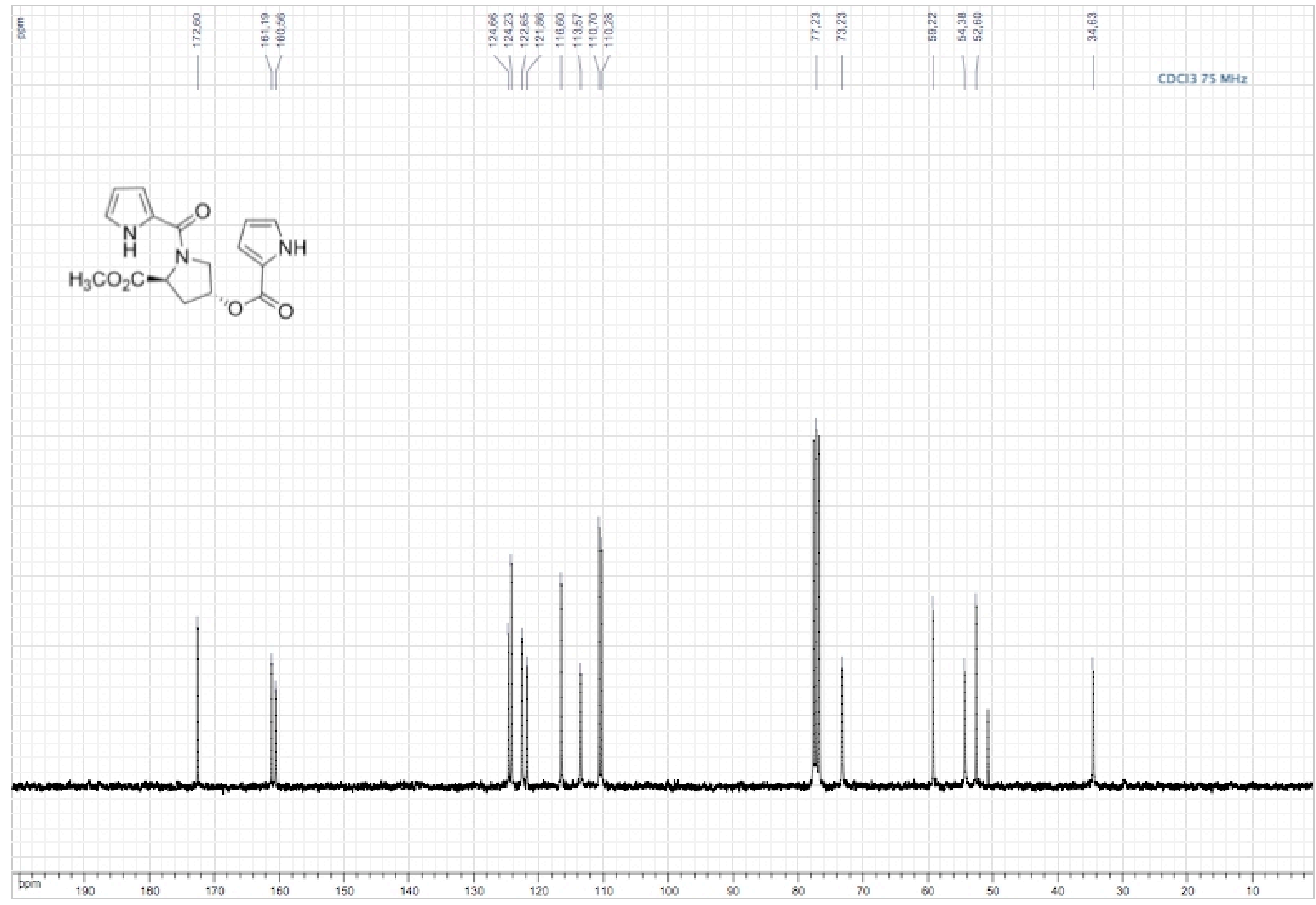


(8R)-8-hydroxy-2-imino-6-(1H-pyrrole-2-carbonyl)-1,3,6-triazaspiro[4.4]nonan-4-one (13), ${ }^{1} \mathrm{H}$ NMR :

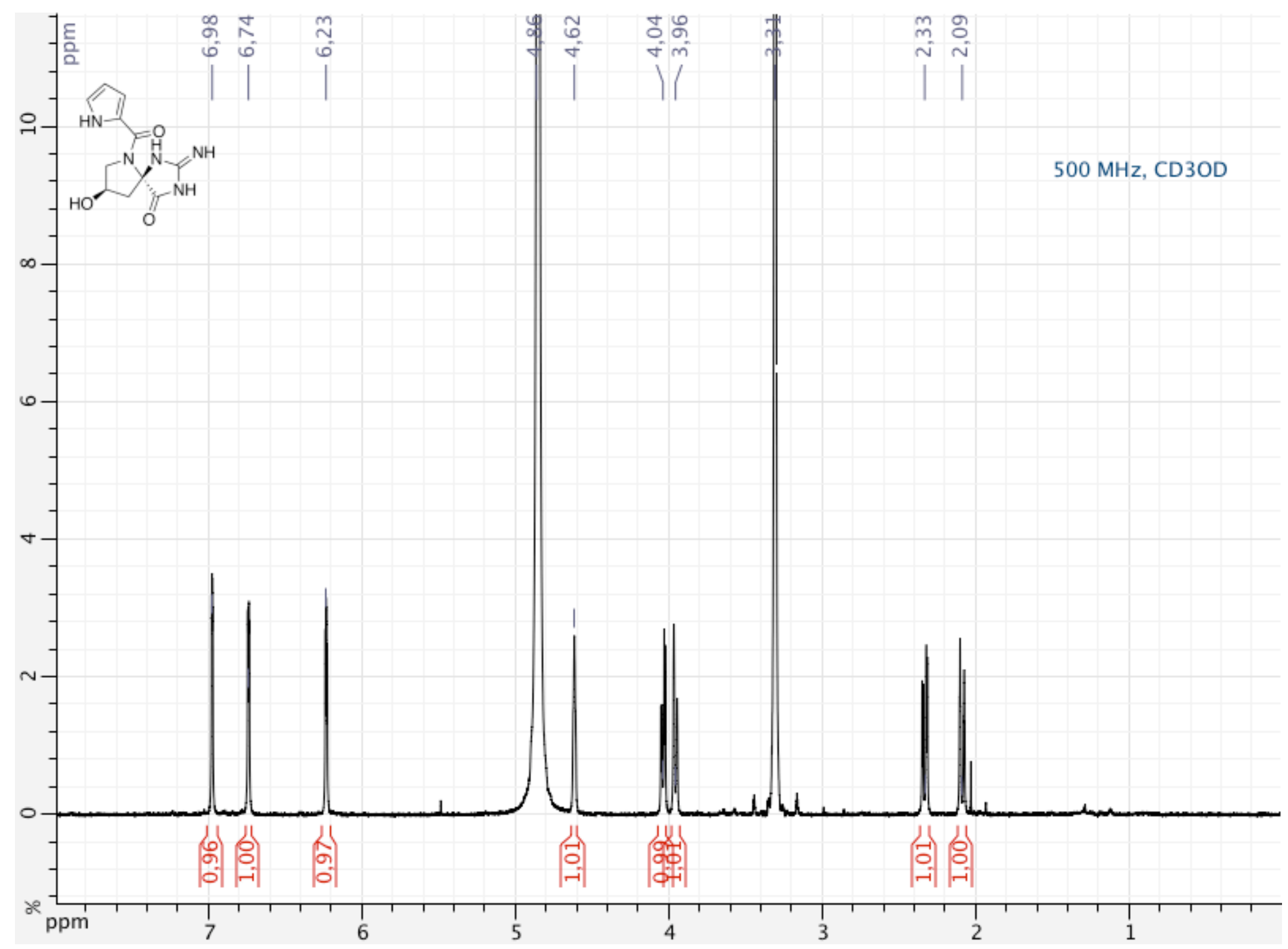


(8R)-8-hydroxy-2-imino-6-(1H-pyrrole-2-carbonyl)-1,3,6-triazaspiro[4.4]nonan-4-one (15), ${ }^{13} \mathrm{C}$ NMR :

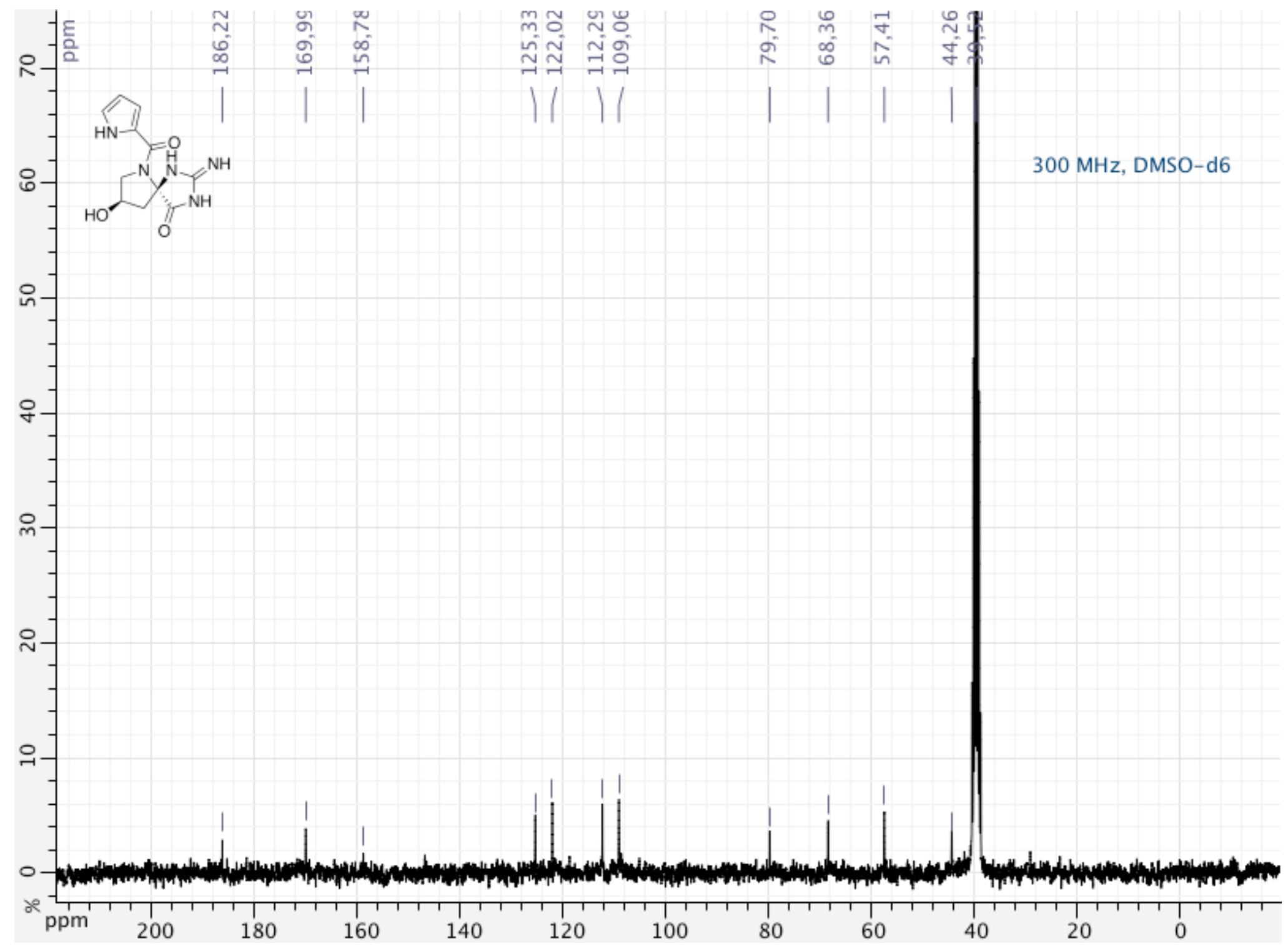


oxazolines $\mathbf{7 a}+\mathbf{1 7} \mathbf{b},{ }^{1} \mathrm{H}$ NMR :

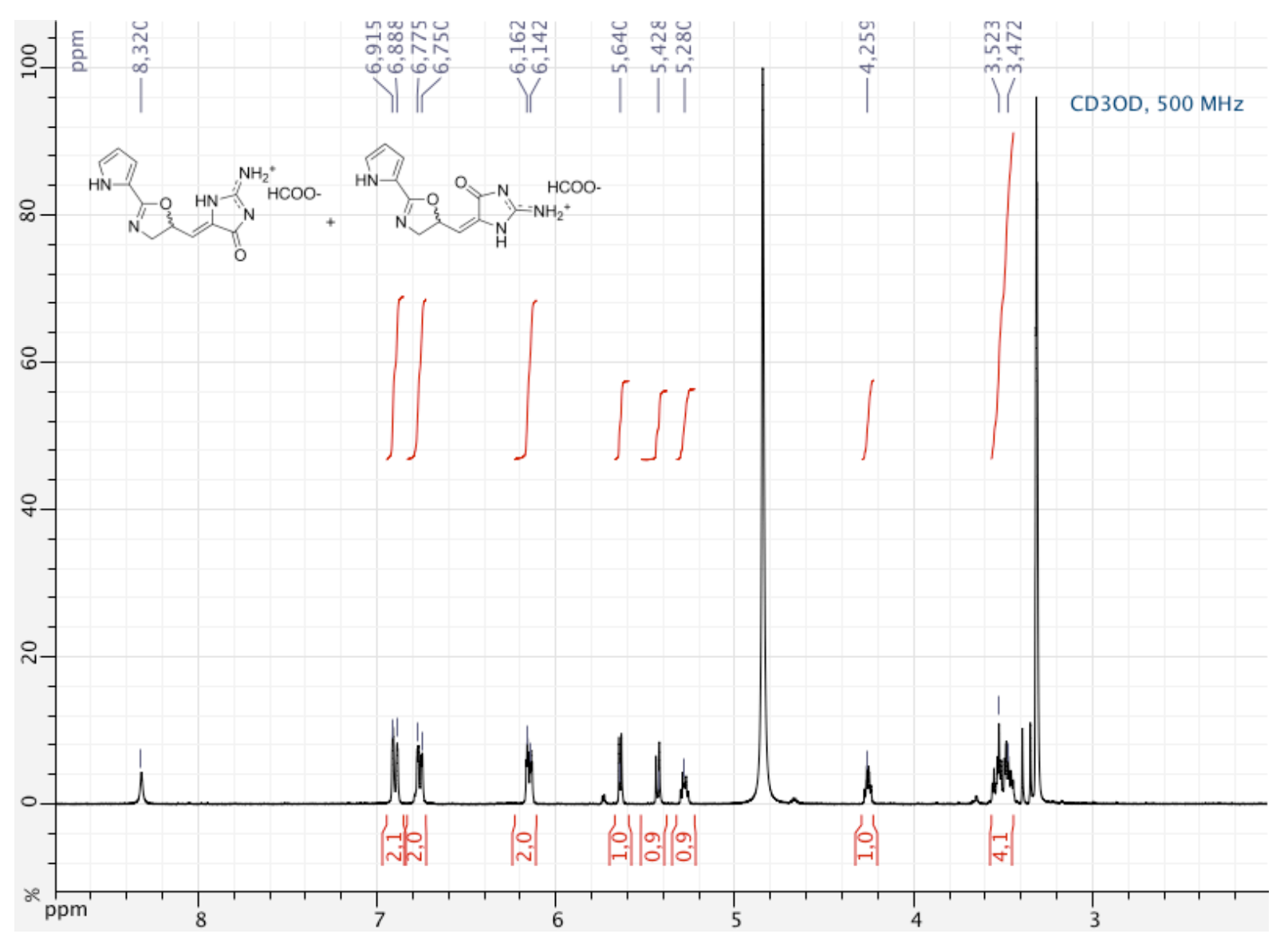


oxazolines $1 \mathbf{7 a}+\mathbf{1 7} \mathbf{b},{ }^{13} \mathrm{C} \mathrm{NMR}$ :

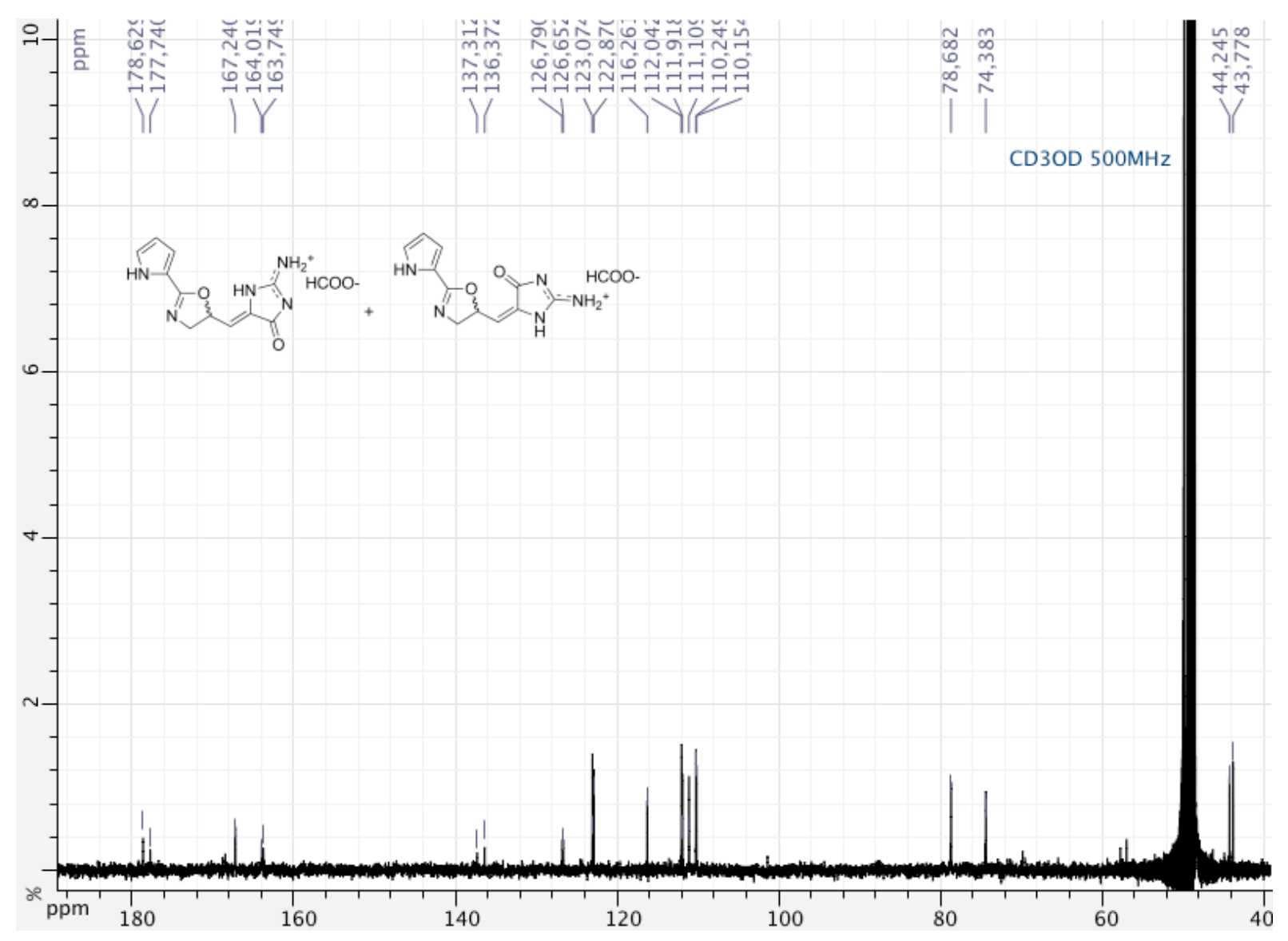

